\title{
ON A REPAIRMAN ASSIGNMENT PROBLEM
}

\author{
Ger Koole \\ CWI
}

Consider a model with $m$ parallel queues, each with its own server, and the same finite capacity $B$. All service times are exponentially distributed with the same rate. Arriving customers have to be assigned to one of the non-empty queues. It is well known that, if the decision is based on the current queue lengths, assigning to the shortest queue is optimal, for a large class of reward functions. Also the arrivals can be quite general, even allowing the arrival stream to depend in some sense on the queue lengths.

Let us now assume that we do not have the current queue lengths available, but only the queue lengths just after the previous arrival. Thus, there is a delay in the arrival of information on the system. It can be proven that with this information delay it is optimal to assign to the queue which was smallest at the previous arrival instant. Note that this queue need not be the smallest anymore (although it is, if we look at it stochastically).

This result, and some variants of it, can be used to analyze several repairman problems. Indeed, consider a system consisting of $m$ groups of parallel components, each with at most $B$ functioning components. The completion of a repair by the repairman corresponds to the arrival of a customer in the routing model described above. It is natural to assume that the repairman :s assigned to a non-functioning component at the beginning of the repair period, representing repair on the spot. This is where the delay comes in; the repairman is assigned to a failed component at the beginning of its repair period, but the repair naturally occurs at the end. If we assume furthermore that in each group at most one component is subject to failure at a time, then this repairman model is completely equivalent with the routing model, and therefore the repairman should be assigned to the group with the smallest number of functioning components. Suitable reward functions are the total number of functioning components, or the probability that each group has at least one functioning component.

Some variants of this model lead to the same result. In the first all functioning components are subject to failure, representing a warm stand-by model. In the second we define the system to be up if each group has at least one functioning component. Only if the system is up components can fail, one at a group. The expected time that the system is up in this cold stand-by model is again maximized by assigning to the group with the smallest number of functioning components. 